Pacific Journal of Mathematics

NOTES ON RELATED STRUCTURES OF A UNIVERSAL 


\section{NOTES ON RELATED STRUCTURES OF A UNIVERSAL ALGEBRA}

\section{WILLIAM A. LAMPE}

The related structures of a universal algebra $\mathfrak{A}$ that are studied here are the subalgebra lattice of $\mathfrak{A}$, the congruence lattice of $\mathfrak{A}$, the automorphism group of $\mathfrak{A}$, and the endomorphism semigroup of $\mathfrak{A}$. Characterizations of these structures known, and $E$. T. Schmidt proved the independence of the automorphism group and the subalgebra lattice. It has been conjectured that the first three of the structures listed above are independent, i.e., that the congruence lattice, subalgebra lattice, and automorphism group are independent. One result in this paper is a proof of a special case of this conjecture. Various observations concerning the relationship between the endomorphism semigroup and the congruence lattice are also in this paper. In the last section a problem of G. Grätzer is solved, namely that of characterizing the endomorphism semigroups of simple unary algebras. (An algebra is simple when the only congruences are the trivial ones.)

The characterizations of the various related structures are as follows: the congruence lattice is an arbitrary algebraic lattice the subalgebra lattice is an arbitrary algebraic lattice; the automorphism group is an arbitrary group; the endomorphism semigroup is an arbitrary semigroup with identity. The "independence of the automorphism group and the subalgebra lattice" is more precisely phrased as: for each pair $\langle\mathbb{B}, \mathbb{R}\rangle$, where $\mathbb{B}$ is a group and $\mathfrak{Z}$ is an algebraic lattice with more than one element, there is an algebra $\mathfrak{A}$ with (S) isomorphic to the automorphism group of $\mathfrak{A}$ and with $\&$ isomorphic to the subalgebra lattice of the same algebra भ. All statements about the independence of related structures will be phrased in this way.

Mentioned above was a proof of a special case of the independence of the triple consisting of the automorphism group, the subalgebra lattice, and the congruence lattice. As a corollary one gets a proof of a special case of the independence of the pair consisting of the automorphism group and the congruence lattice. E. T. Schmidt published what was supposed to be a proof of the independence of this pair of structures. But, his proof [10] was incorrect. (See e.g. Exercise 31 of chapter 2 of [2]). The author has just completed a proof of the independence of this pair [8].

The terminology essentially conforms to that in [2]. $\omega\left(\right.$ or $\left.\omega_{A}\right)$ will denote the equality relation on the set $A$, and $\iota\left(\right.$ or $\left.\iota_{A}\right)$ will denote 
the total relation. $\Theta\left(a_{0}, a_{1}\right)$ will represent the smallest congrucence collapsing $a_{0}$ and $a_{1} . \mathfrak{Q}=\langle L, \wedge, \vee\rangle$ will denote a lattice. $\mathfrak{E}(\mathfrak{R})=$ $\langle\mathscr{C}(\mathfrak{H}) ; \cong\rangle$ will denote the congruence lattice of $\mathfrak{A}$. $\mathscr{S}(\mathfrak{A})=\langle\mathscr{S}(\mathfrak{A})$; $\subseteq\rangle$ will denote the subalgebra lattice of $\mathfrak{X} . \mathfrak{S}(\mathfrak{U})=\langle G(\mathfrak{U})$; $\circ\rangle$ will denote the automorphism group of $\mathfrak{A}$. $(\mathfrak{F}(\mathfrak{X})=\langle E \mathfrak{Q})$; 0$\rangle$ will denote the endomorphism semigroup of $\mathfrak{A}$.

An important algebra for dealing with endomorphism semigroup and automorphism group problems is the algebra of left multiplications $\mathfrak{2 ( S )}$ of the semigroup $\mathfrak{S}$. The operations are all left multiplication maps and the endomorphisms are all right multiplication maps. As in Cayley's Theorem, the semigroup of right multiplications of $\mathfrak{S}$ is isomorphic to $\mathfrak{S}$.

Many of the details of the proofs which are left out can be found in the author's dissertation [6]. The various characterizations mentioned above can be found in [1], [2], [3]. E. T. Schmidt's result on the independence of the automorphism group and subalgebra lattice is found in [11].

1. The property restricting the representation of $\left\langle\mathbb{E}, \mathbb{R}_{0}, \mathbb{R}_{1}\right\rangle$ as $\langle\mathfrak{S}(\mathfrak{U}), \mathfrak{S}(\mathfrak{U}), \mathfrak{S}(\mathfrak{U})\rangle$.

Let $\mathfrak{A}=\langle A ; F\rangle$ be an algebra. The lattice $\mathfrak{Z}$ is assumed to be an algebraic lattice. Let $a \in L$, and let $\left(x_{i} \mid i \in I\right)$ be a family of elements of $L$.

Essentially the property mentioned in the heading is: there exist $a_{0}, a_{1} \in A$ such that for any $x \neq a_{0}$ and for any congruence $\Theta$, if $a_{0} \equiv$ $x(\Theta)$, then $a \equiv a_{1}(\Theta)$. We will give a generalization of this property and a property of the congruence lattice equivalent to the more general property. Also, the class of algebraic lattices having the equivalent property will be discussed.

Let $a_{0}, a_{1} \in A$ with $a_{0} \neq a_{1}$.

(**) There exists a partition $\left\{A_{0}, A_{1}\right\}$ of $A$ such that $a_{i} \in A_{i}$ and for any $\langle x, y\rangle \in A_{0} \times A_{1}, \Theta\left(a_{0}, a_{1}\right) \leqq \Theta(x, y)$.

(*) If $a \leqq \bigvee\left(x_{i} \mid i \in I\right)$, then $a \leqq x_{i}$ for some $i$.

Notice that the originally stated condition is a special case of $\left({ }^{*}\right)$ where $A_{0}=\left\{a_{0}\right\}$. Obviously, if an element $a$ of $\mathfrak{L}$ has property $(*)$, then $a$ is complete-join irreducible. Also, $a$ has property $\left(^{*}\right)$ if and only if $a$ 's dual ideal is completely prime.

Proposition 1. If property $\left(^{* *}\right)$ is satisfied for $\left\langle a_{0}, a_{1}\right\rangle$, then $\Theta\left(a_{0}, a_{1}\right\rangle$ satisfies property $\left(^{*}\right)$ in the congruence lattice of $\mathfrak{A}$. 
REMARK. This statement was first observed by G. Grätzer.

Proof. Suppose that $\left(\Phi_{i} \mid i \in I\right)$ is a family of congruences and that $\Theta\left(a_{0}, a_{i}\right) \subseteq \mathrm{V}\left(\Phi_{i} \mid i \in I\right)$. There exists a sequence $a_{0}=z_{0}, \cdots, z_{n}$, $=a_{1}$, with $z_{j} \in A$ such that $z_{j} \equiv z_{j+1}\left(\Phi_{i_{j}}\right)$ for some $i_{j} \in I$. Since $a_{0} \in$ $A_{0}, a_{1} \in A_{1}$, and $\left\{A_{0}, A_{1}\right\}$ is a partition of $A$, there is a $k$ such that $z_{k} \in$ $A_{0}$ and $z_{k+1} \in A_{1}$. So $\Theta\left(a_{0}, a_{1}\right) \subseteq \Theta\left(z_{k}, z_{k+1}\right) \subseteq \Phi_{i_{k}}$.

Proposition 2. If there is a congruence $\Theta$ different from $\omega$ having property $\left(^{*}\right)$, then $\Theta=\Theta\left(a_{0}, a_{1}\right)$ for some $a_{0}, a_{1}$ in $A$ with $a_{0} \neq a_{1}$ and property (**) is satisfied for $\left\langle a_{0}, a_{1}\right\rangle$.

Proof. Always $\Theta=\mathrm{V}(\Theta(x, y) \mid x \equiv y(\Theta))$. Since $\Theta$ has property $\left(^{*}\right)$, $\Theta=\Theta(x, y)$ for some $x, y \in A$. Fix $a_{0}, a_{1}$ such that $\Theta=\Theta\left(a_{0}, a_{1}\right)$. Set

$$
\begin{aligned}
& B_{0}=\left\{x \mid \Theta\left(x, a_{0}\right) \nsupseteq \Theta\left(a_{0}, a_{1}\right)\right\}, \\
& B_{1}=\left\{y \mid \Theta\left(y, a_{1}\right) \nsupseteq \Theta\left(a_{0}, a_{1}\right)\right\}, \\
& B_{2}=\left\{z \mid \Theta\left(a_{0}, z\right)=\Theta\left(a_{1}, z\right)\right\} .
\end{aligned}
$$

Set $A_{0}=B_{0}$ and $A_{1}=B_{1} \cup B_{2}$. It follows that $A_{0} \cap A_{1}=\varnothing$. Clearly, $a_{0} \in A_{0}$ and $a_{1} \in A_{1}$. Also $A=A_{1} \cup A_{2}$.

Let $x_{0} \in A_{0}$ and $x_{1} \in A_{1}$ and consider $\Theta\left(x_{0}, x_{1}\right)$. First suppose that $x_{1} \in B_{1}$. Thus, $\Theta\left(x_{0}, a_{0}\right) \nsupseteq \Theta\left(a_{0}, a_{1}\right)$ and $\Theta\left(x_{1}, a_{1}\right) \nsupseteq \Theta\left(a_{0}, a_{1}\right)$. Now, since $\Theta\left(a_{0}, a_{1}\right) \leqq \Theta\left(x_{0}, a_{0}\right) \vee \Theta\left(x_{0}, x_{1}\right) \vee \Theta\left(x_{1}, a_{1}\right)$ and since $\Theta\left(a_{0}, a_{1}\right)$ has $\left(^{*}\right)$, we have that $\Theta\left(a_{0}, a_{1}\right) \subseteq \theta\left(x_{0}, x_{1}\right)$. Now suppose that $x_{1} \in B_{2}$. So $\Theta\left(a_{0}, a_{1}\right) \subseteq$ $\Theta\left(a_{0}, x_{1}\right) \subseteq \Theta\left(a_{0}, x_{0}\right) \vee \Theta\left(x_{0}, x_{1}\right)$, and thus, $\Theta\left(a_{0}, a_{1}\right) \subseteq \Theta\left(x_{0}, x_{1}\right)$.

Combining these two propositions with the congruence lattice characterization theorem, we get the following statement.

Proposition 3. If $\mathrm{Q}$ is an algebraic lattice, the following are equivalent:

(i) there exists $a \neq 0, a \in L$, such that a has property $\left({ }^{*}\right)$;

(ii) there exists an algebra $\mathfrak{U}=\langle A ; F\rangle$ with $\mathbb{5}(\mathfrak{Z})$, the congruence lattice of $\mathfrak{A}$, isomorphic to $\mathfrak{R}$, and there are $a_{0}, a_{1} \in A, a_{0} \neq$ $a_{1}$, such that $\left({ }^{* *}\right)$ is satisfied for $\left\langle a_{0}, a_{1}\right\rangle$;

(iii) for any algebra $\mathfrak{U}=\langle A ; F\rangle$ with $\mathfrak{S}(\mathfrak{R})$ isomorphic to $\mathfrak{R}$, there are $a_{0}, a_{1} \in A, a_{0} \neq a_{1}$, such that $\left(^{* *}\right)$ is satisfied for $\left\langle a_{0}, a_{1}\right\rangle$.

Let $\mathscr{K}$ be the class of algebraic lattices having an $a \neq 0$ with property $\left(^{*}\right)$. Several simple observations can be made. The five element modular non-distributive lattice is not in $\mathscr{K}$ since none of the dual ideals generated by a nonzero element is prime. Every distributive algebraic lattice with a complete-join irreducible element is in $\mathscr{K}$. If $\Omega_{1}$ and $\mathbb{\Omega}_{2}$ are algebraic lattices, then $\mathfrak{\Omega}_{1}+\mathfrak{\Omega}_{2} \in \mathscr{K}$ (where + 
denotes ordinal sum). (The zero of $\Omega_{2}$ is a nonzero element in $\Omega_{1}+$ $\mathfrak{L}_{2}$ having $\left(^{*}\right)$ ). Every algebraic lattice $\mathfrak{Z}$ is both a complete sublattice of and a homomorphic image of a member of $\mathscr{K}$ since $\mathbb{C}_{1}+\mathbb{L} \in \mathscr{K}$. $\left(\mathbb{C}_{n}\right.$ denotes the $n$-element chain.) Also, observe that for a family $\left(\Omega_{i} \mid i \in I\right)$ of algebraic lattices, $\Pi\left(\Omega_{i} \mid i \in I\right) \in \mathscr{K}$ if and only if there exists at least one $j \in I$ with $\mathfrak{\Omega}_{j} \in \mathscr{K}$.

2. The construction for representing $\left\langle\left(\mathbb{S}, \mathfrak{R}_{0}, \mathfrak{R}_{1}\right\rangle\right.$ as $\langle(\mathbb{S}(\mathfrak{Q}), \mathfrak{S}(\mathfrak{Q}), \mathfrak{C}(\mathfrak{W})\rangle$. First we need some notation. Let $\mathfrak{U}=\langle A ; F\rangle$ be an algebra and $X \subseteq A$. Set $F(\mathfrak{H}, X)=\left\{\varphi \mid \varphi\right.$ is an endomorphism of $\mathfrak{A},\{x\}=x \varphi^{-1}$ for all $x \in X\}$, and set $\mathfrak{F}(\mathfrak{A}, X)=\langle F(\mathfrak{A}, X) ; \circ\rangle$. In other words, an endomorphism $\varphi$ is in $F(\mathfrak{A}, X)$ if $(A-X) \varphi \subseteq A-X$ and $x \varphi=x$ for $x \in X$. Clearly, $\mathfrak{F}(\mathfrak{A}, X)$ is a nonempty semigroup with identity.

$\mathscr{S}(\mathfrak{A})$ is the subalgebra system of $\mathfrak{A}$. Recall that $\mathfrak{S}(\mathfrak{Z})$ is the subalgebra lattice, that $\mathfrak{S}(\mathfrak{N})$ is the congruence lattice, and that $\mathfrak{F}(\mathfrak{H})$ is the endomorphism semigroup.

TheOREM 1. Suppose that $\mathfrak{A}$ and $\mathfrak{B}$ are algebras, that $\mathfrak{A}$ is simple, that there is a $U \subseteq A,|U|=2, U \subseteq D$ for every $D \in \mathscr{S}(\mathfrak{X})$, and that there is an $\left\langle a_{0}, a_{1}\right\rangle \in B^{2}$ with $a_{0} \neq a_{1}$ for which property (**) is satisfied. There exists an algebra $\mathfrak{X}^{\prime}$ such that:

(i) $\mathfrak{S}\left(\mathfrak{U}^{\prime}\right)$ is isomorphic to $\mathfrak{S}(\mathfrak{R})$;

(ii) $\mathfrak{C}\left(\mathfrak{H}^{\prime}\right)$ is isomorphic to $\mathfrak{C}(\mathfrak{B})$;

(iii) $\mathfrak{F}\left(\mathfrak{U}^{\prime}\right)$ is isomorphic to $\mathfrak{F}(\mathfrak{H}, U)$.

Proof. Let $\mathfrak{U}=\langle A ; F\rangle$ and $\mathfrak{B}=\langle B ; G\rangle$ and $U=\left\{u_{0}, u_{1}\right\}$ and let $\left\langle a_{0}, a_{1}\right\rangle \in B^{2}$ have $\left({ }^{* *}\right)$ and let $a_{0} \neq a_{1}$. Assume that $A$ and $B$ are disjoint. For each $x \in B \cup U$ define a nullary operation $f_{x}$ whose value is $x$. Let $\left\{A_{0}, A_{1}\right\}$ be a partition of $B$ for satisfying (**). Define four unary operations as follows:

$$
\begin{aligned}
& g_{1}(x)=\left\{\begin{array}{l}
u_{0}, x \in A \cup A_{0} \\
u_{1}, x \in A_{1}
\end{array} ;\right. \\
& g_{2}(x)=\left\{\begin{array}{l}
a_{0}, x=u_{0} \\
a_{1}, \text { otherwise }
\end{array} ;\right. \\
& g_{3}(x)=\left\{\begin{array}{l}
a_{0}, x \in A \\
a_{1}, x \in B
\end{array} ;\right. \\
& g_{4}(x)=\left\{\begin{array}{l}
x, x \in A-U \\
u_{0}, x \in B \cup\left\{u_{1}\right\} \\
a_{0}, x=u_{0}
\end{array}\right.
\end{aligned}
$$

For $x \in A^{\prime}$, set $\hat{x}=x$ if $x \in B$ and set $\hat{x}=a_{0}$ if $x \in A$. Let $x_{i} \in A^{\prime}$. 
Extend the operations of $F$ and $G$ to $A^{\prime}$ as follows: For $f \in G$ set $f\left(a_{0}, \cdots, a_{n-1}\right)=f\left(\hat{a}_{0}, \cdots, \hat{a}_{n-1}\right)$. For $f \in F$, if all $a_{i} \in A$, then keep the value of $f$ in $\mathfrak{A}$, and set $f\left(a_{0}, \cdots, a_{n-1}\right)=u_{0}$, otherwise. Set $F^{\prime}=F \dot{\cup} G \dot{\cup}\left\{f_{x} \mid x \in B \dot{\cup} U\right\} \dot{\cup}\left\{g_{i} \mid i=1,2,3,4\right\}$. Set $\mathfrak{X}^{\prime}=\left\langle A^{\prime} ; F^{\prime}\right\rangle$.

For each $D \in \mathscr{S}(\mathfrak{N})$, set $\bar{D}=D \cup B$. For each $\varphi \in F(\mathfrak{A}, U)$, define $\bar{\varphi}$ by letting $x \bar{\varphi}=x \varphi$ if $x \in A$ and $x \bar{\varphi}=x$ if $x \in B$. For $\Theta \in \mathscr{C}(\mathfrak{V})$ define $\Theta^{*}$ by letting $\Theta^{*}=\omega_{A}$, the equality relation on $A$, if $\Theta\left(a_{0}, a_{1}\right) \nsubseteq \Theta$ and $\Theta^{*}=\iota_{A} \cup\left\{\langle x, b\rangle \mid x \in A, b \equiv a_{0}(\Theta)\right\} \cup\left\{\langle b, x\rangle \mid x \in A, b \equiv a_{0}(\Theta)\right\}$ in case $\Theta\left(a_{0}, a_{1}\right) \subseteq \Theta$. Now set $\bar{\Theta}=\Theta \cup \Theta^{*}$. To complete the proof one shows that $D \rightarrow \bar{D}, \varphi \rightarrow \bar{\varphi}$, and $\Theta \rightarrow \bar{\Theta}$ are isomorphisms. The lengthy, but routine, calculations are left to the reader.

In the proof above the operation $g_{4}$ guarantees that an endomorphism $\sigma$ of $\mathfrak{X}^{\prime}$ has the property that $A \sigma \subseteq A$. The operations $g_{1}, g_{2}, g_{3}$ guarantee that $a_{0} \equiv a_{1}$ iff $u_{0} \equiv u_{1}$ iff $a_{0} \equiv a_{1} \equiv u_{0} \equiv u_{1}$. That $\mathfrak{X}$ is simple guarantees that if $x, y \in A$ and $x \neq y$ and $x \equiv y$ then $x \equiv u_{0} \equiv$ $u_{1}$. Finally $g_{3}$ guarantees that if $x \in A$ and $y \in B$ and $x \equiv y$ then $a_{0} \equiv a_{1}$.

\section{Representing $\left\langle\mathbb{S}, \mathfrak{Z}_{0}, \mathfrak{Q}_{1}\right\rangle$ as $\langle\mathbb{S}(\mathfrak{H})$, $\mathfrak{S}(\mathfrak{U})$, $\mathfrak{S}(\mathfrak{H})\rangle$.}

LEMmA 1. If $\mathfrak{X}=\langle A ; F\rangle$ is an algebra, then there is an algebra $\mathfrak{X}^{\prime}=\langle A ; F\rangle$ such that:

(i) $\mathfrak{U}^{\prime}$ is simple;

(ii) $D$ is a subalgebra of $\mathfrak{A}$ iff $D$ is a subalgebra of $\mathfrak{X}^{\prime}$;

(iii) $E(\mathfrak{H})=\{\varnothing \mid \varphi \in E(\mathfrak{U}), \varphi$ is $1-1$ or $\varphi$ is constant $\}$.

REMARks. Roughly (iii) says $E\left(\mathfrak{U}^{\prime}\right)$ is as big as is possible given (i) and (ii).

Suppose $\subseteq$ is a semigroup in which every element is right cancellative or a right zero. Every endomorphism of $\mathbb{R}(\mathfrak{S})$ is $1-1$ or constant. By applying this lemma to $\mathfrak{L}(\mathfrak{S})$ we get an easier proof that $\left\langle\mathfrak{S}_{;} \mathfrak{S}_{2}\right\rangle$ is representable. (See [3].)

Proof. Add an additional operation $g$ defined as follows:

$$
g(x, y, u, v)=\left\{\begin{array}{l}
u, \text { if } x \neq y \\
v, \text { if } x=y
\end{array}\right.
$$

Suppose $x \neq y$ and $\Theta$ is any congruence of $\mathfrak{X}^{\prime}$ with $x \equiv y(\theta)$. Let $u, v \in A$. Thus, $u=g(x, y, u, v) \equiv g(y, y, u, v)=v(\Theta)$. So $\Theta=\iota$, and (i) is established.

The rest is routine. 
The operation used in the above lemma was used in [5] in a different context, but in each case the purpose of the operation is to "fill out" subalgebras in a direct power. This 4-ary function is equivalent to the ternary discriminator function [12] [9] in that each can be expressed as a polynomial in the other.

A modification of the above 4-ary function is used in Lemma 6 . It does not appear that the modified 4-ary function is equivlent to a ternary function.

LEMMA 2. If $\mathfrak{A}$ is any algebra, then there is an algebra $\mathfrak{H}^{\prime}=$ $\left\langle A^{\prime} ; F^{\prime}\right\rangle$ and $U \subseteq A^{\prime}$ with $|U|=2$ such that:

(i) $\mathfrak{S}(\mathfrak{U})$ is isomorphic to $\mathfrak{S}\left(\mathfrak{U}^{\prime}\right)$;

(ii) $U \subseteq D$ for all $D \in \mathscr{S}\left(\mathfrak{U}^{\prime}\right)$;

(iii) $\mathfrak{F}(\mathfrak{U}, U)$ is isomorphic to $\mathfrak{S}(\mathfrak{U})$.

Proof. Add two elements $u_{0}, u_{1}$. Let $u_{0}$ and $u_{1}$ each be the value of a nullary operation. Extend every operation $f$ of $\mathfrak{A}$ by setting $f\left(x_{0}, \cdots, x_{n-1}\right)=u_{0}$ if $x_{j} \in U$. The rest is obvious.

The next lemma is a theorem due to E. T. Schmidt [11]. Recall that $\mathbb{B}(\mathfrak{A})$ is the automorphism group of $\mathfrak{A}$.

LEMMA 3. If \&S is any group and $\&$ is any algebraic lattice with $|L|>1$, then there is an algebra $\mathfrak{A}$ with $\mathbb{B}$ isomorphic to $\mathbb{S}(\mathfrak{A})$ and

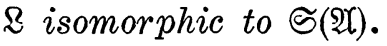

THEOREM 2. If \&S is any group, if $\mathfrak{\Omega}_{0}$ and $\mathfrak{\Omega}_{1}$ are algebraic lattices such that $\left|L_{0}\right|>1$, and if there is an $a \neq 0, a \in L_{1}$, with property $\left({ }^{*}\right)$, then there is an algebra $\mathfrak{O}$ such that:

(i) (S) is isomorphic to $\mathbb{S}(\mathfrak{2})$;

(ii) $\mathfrak{\Omega}_{0}$ is isomorphic to $\mathfrak{S}(\mathfrak{2})$;

(iii) $\mathfrak{Q}_{1}$ is isomorphic to $\mathfrak{E}(\mathfrak{Z})$.

REMARKS. A best possible representation theorem would, of course, have the restriction that $\left|L_{1}\right|>1$. Also, if $\left|L_{0}\right|=1$, then it is necessary that $|G|=1$. Of course any triple of the form $\left\langle 1, \mathfrak{E}_{1}, \mathbb{R}\right\rangle$ is representable. $\left(\mathfrak{\mho}_{1}\right.$ is the one element chain.)

Proof. Let $\mathfrak{B}$ be the algebra given by Lemma 3 when applied to $\left(\mathfrak{S}\right.$ and $\mathfrak{R}_{0}$. Let $\mathfrak{B}^{\prime}$ be the algebra given by Lemma 2 applied to $\mathfrak{B}$. Let $\mathfrak{B}^{\prime \prime}$ be the algebra given by Lemma 1 . Let $\mathbb{E}$ be the algebra constructed in the proof of the congruence lattice characterization theorem [2], [4] or [7]. Let $\mathfrak{X}$ be the algebra given by Theorem 1 when applied to $\mathfrak{B}^{\prime \prime}$ and $\mathfrak{C}$. The rest is routine. 
COROLlaRY 1. If (S) is any group and $\mathbb{2}$ is any algebraic lattice with $a \neq 0, a \in L$, having property $\left({ }^{*}\right)$, then there is an algebra $\mathfrak{X}$ with (S) isomorphic to (S)(I) and $\mathbb{2}$ isomorphic to $\mathfrak{S}(\mathfrak{U})$.

COROLlaRY 2. If $\mathfrak{\Omega}_{0}$ is any algebraic lattice and $\mathfrak{\Omega}_{1}$ is an algebraic lattice with an $a \neq 0\left(a \in L_{1}\right)$ such that a has property $\left({ }^{*}\right)$, then there is an algebra $\mathfrak{A}$ with $\mathfrak{S}(\mathfrak{U})$ isomorphic to $\mathfrak{\Omega}_{0}$ and $\mathfrak{S}(\mathfrak{U})$ isomorphic to $\mathfrak{\Omega}_{1}$.

4. Necessary conditions for $\langle\subseteq, \&\rangle$ to be representable as $\langle\mathfrak{S}(\mathfrak{U}), \mathfrak{S}(\mathfrak{U})\rangle$. Recall that if $\mathfrak{S}$ is a semigroup, $\mathfrak{L}(\mathfrak{S})$ is the algebra of left multiplications of $\mathfrak{S}$. $\mathfrak{A}=\langle A ; F\rangle$ is some universal algebra. The basic thing established in this section is a relationship between $\mathfrak{C}\left(\mathfrak{Q}(\mathfrak{F}(\mathfrak{Q}))\right.$ ) and $\mathfrak{F}(\mathfrak{U})$. If $\varphi$ is an endomorphism, then set $x \equiv y\left(\varepsilon_{\varphi}\right)$ iff $x \varphi=y \varphi . \quad \varepsilon_{\varphi}$ is a congruence.

Let $\mathfrak{S}=\langle S ; \cdot\rangle$ be a semigroup with identity, and let $x, s \in S$. The right multiplication map for $s$ is defined by $x \rho_{s}=x s$.

Thus, if $\varphi \in E(\mathfrak{X})$, then we have the congruence $\varepsilon_{\varphi}$ on $\mathfrak{Y}$ and the mapping $\rho_{\varphi}$ on $E(\mathfrak{Q})$. So we have the equivalence relation $\varepsilon_{\rho_{\varphi}}$ on $E(\mathfrak{H})$. Observe that since $\rho_{\varphi}$ is an endomorphism of $\mathbb{L}(\mathfrak{F}(\mathfrak{Z}))$, $\varepsilon_{\rho_{\varphi}}$ is a congruence of $\mathfrak{L}(\mathfrak{S}(\mathfrak{U}))$.

The proof of the next lemma involves only routine calculations.

LEMmA 4. If $\varepsilon_{\psi}=\bigcap\left(\varepsilon_{\varphi_{i}} \mid i \in I\right)$, then $\varepsilon_{\rho_{\psi}}=\bigcap\left(\varepsilon_{\rho_{\varphi_{i}}} \mid i \in I\right)$.

COROLlaRY. $\varepsilon_{\varphi} \rightarrow \varepsilon_{\rho_{\varphi}}$ is a mapping, and this mapping preserves arbitrary existing meets. In particular, it is order preserving.

This mapping need not be $1-1$.

LEMMA 5. If $\varepsilon_{\rho_{\varphi}}=\iota$ ( $\varphi$ is a right zero), then $\varepsilon_{\psi} \leqq \varepsilon_{\varphi}$ for every endomorphism $\psi$.

\section{Proof. Trivial.}

$\varepsilon_{\rho_{\varphi}}$ can be $\iota$ and $\varepsilon_{\varphi}$ need not be $\iota . \varepsilon_{\rho_{\varphi}}=\iota$ means $\varphi$ is a right zero in $(\mathfrak{F}(\mathfrak{U})$, but $\varphi$ need not be a constant map. But $\varphi$ is a constant map iff $\varepsilon_{\varphi}=\imath$. On the other hand, if $\varepsilon_{\varphi}=\iota$, then $\varepsilon_{\rho_{\varphi}}=\iota$ (i.e., if $\phi$ is a constant map, then $\varphi$ is a right zero). Also, there is a $\varphi$ with $\varepsilon_{\varphi}=\omega$ and $\varepsilon_{\rho_{\varphi}}=\omega$ (the identity map).

To summarize we state the following theorem.

Theorem 3. Suppose $\mathfrak{S}=\langle S ; \cdot\rangle$ is a semigroup with identity and $\mathfrak{L}=\langle L ; \vee, \wedge\rangle$ is an algebraic lattice. Set $\mathscr{C}_{\mathcal{C}}=\left\{\varepsilon_{\rho_{s}} \mid s \in S\right\}$. If 
$\langle\subseteq, \mathbb{Q}\rangle$ is representable, then there is a subset $H$ of $L$ and there are two mappings $\alpha$ from $S$ onto $H$ and $\beta$ from $H$ onto $\mathscr{K}$ such that the following hold:

(i) $(s \alpha) \beta=\varepsilon_{\rho_{s}}$ for all $s \in S$;

(ii) $\beta$ preserves arbitrary existing meets;

(iii) if $\varepsilon_{\rho_{s}}=\iota$, then $s \alpha$ is the maximum element of $H$ and $\left|€ \beta^{-1}\right|=1$

(iv) $0 \in H($ and $0 \beta=\omega)$;

(v) if $1 \in H$, then $\iota \in \mathscr{K}$.

CoROLlaRy. If $\left\langle\mathfrak{S}, \mathfrak{F}_{n}\right\rangle$ is representable and $\mathfrak{\Im}_{n}$ is the n-element chain, then $\mathscr{K} \cup\{c\}$ is a chain of length $\leqq n$.

5. More on the class of representable pairs. Throughout this section, $\widetilde{S}=\langle S ; \cdot\rangle$ will be a semigroup with identity and $\mathbb{Q}$ will be an algebraic lattice. The ordinal sum of the lattices will be denoted by.$+ \mathfrak{F}_{n}$ is the $n$ element chain. $\mathfrak{A}=\langle A ; F\rangle$ is an algebra.

In the preceding section, a necessary condition for $\langle\subseteq, \&\rangle$ to be representable as $\langle\mathfrak{S}(\mathfrak{U}), \mathfrak{S}(\mathfrak{V})\rangle$ was given. Roughly the condition states that $\mathfrak{S}$ gives a lower bound on the cardinality of $L$, namely, $|\mathscr{K}|$, and an upper bound on the meet struture of part of 2 . This suggests that one could take a representable pair and expand the lattice and expect the result to be a representable pair. A few such expansions are given here.

Sort of a multiplication formula for members of the class of all representable pairs is given.

One could question whether or not there exist a semigroup with identity and an algebraic lattice which are in some vague sense completely "incompatible." Theorem 4 gives a negative answer.

First we will state the theorems, and then we will give sketches of their proofs.

THEOREM 4. If $\subseteq$ is any semigroup with identity and $\&$ is any algebraic lattice, then there is an algebra $\mathfrak{A}$ with $\subseteq$ isomorphic to $\mathfrak{F}(\mathfrak{H})$ and $\mathfrak{\&}$ isomorphic to a sublattice of $\mathfrak{C}(\mathfrak{Y})$.

This follows from Theorem 7.

THEOREM 5. If $\langle\mathfrak{S} ; \mathfrak{R}\rangle$ is representable, then $\left\langle\mathfrak{S} ; \mathfrak{R}+\mathfrak{C}_{1}\right\rangle$ is representable.

CoRoLlaRY 1. If $\left\langle\mathfrak{S}_{;} \mathfrak{C}_{k}\right\rangle$ is representable, then $\left\langle\mathfrak{S}_{;} \mathfrak{S}_{n}\right\rangle$ is repre-. sentable for any $n \geqq k$.

COROLlaRY 2. If every member of $\mathfrak{S}$ is right cancellative or is 
a right zero and $n \geqq 2$, then $\left\langle\subseteq, \mathfrak{S}_{n}\right\rangle$ is representable.

See [3], or see the remarks after Lemma 1.

THEOREM 6. If $\left\langle\mathfrak{S}_{0}, \mathfrak{\Omega}_{0}\right\rangle$ is representable and $\mathfrak{\Omega}_{1}$ is any algebraic lattice, then both $\left\langle\mathfrak{S}_{0}, \mathfrak{\Omega}_{0}+\mathfrak{\Omega}_{1}+\mathfrak{S}_{1}\right\rangle$ and $\left\langle\mathfrak{S}_{0}, \mathfrak{\Omega}_{1}+\mathfrak{\Omega}_{0}+\mathfrak{C}_{1}\right\rangle$ are representable.

THEOREM 7. If $\left\langle\mathfrak{S}_{0}, \mathfrak{\Omega}_{0}\right\rangle$ is representable and $\mathfrak{\Omega}_{1}$ is any algebraic lattice, then $\left\langle\mathfrak{S}_{0},\left(\mathfrak{S}_{0} \times \mathfrak{\Omega}_{1}\right)+\mathfrak{S}_{1}\right\rangle$ is representable.

This is a special case of Theorem 8 .

THEOREM 8. If $\left\langle\mathfrak{S}_{0}, \mathfrak{\Omega}_{0}\right\rangle$ and $\left\langle\mathfrak{S}_{1}, \mathfrak{Q}_{1}\right\rangle$ are representable, then $\left\langle\widetilde{S}_{0} \times \mathfrak{S}_{1},\left(\mathfrak{\Omega}_{0} \times \mathfrak{\Omega}_{1}\right)+\mathfrak{S}_{1}\right\rangle$ is representable.

Note that each of the "+ $\widetilde{C}_{1}$ "'s gives us a nonzero element in the resulting lattice that has property $\left({ }^{*}\right)$. (See §1.)

In Theorem 6 one can easily do without the " $+\mathfrak{C}_{1}$ " in the first pair (i.e., one can show $\left\langle\mathfrak{S}_{0}, \mathbb{\Omega}_{0}+\mathfrak{R}_{1}\right\rangle$ is representable) in case $\mathfrak{\Omega}_{1}$ already had a non-zero element satisiying property $\left(^{*}\right)$. A similar comment can be made for the other pair in Theorem 6 in case $\mathfrak{Z}_{0}$ already had a non-zero element satisfying (*). To do the same for Theorem 7 or 8 would seem to require that both $\mathfrak{s}_{0}$ and $\mathfrak{心}_{1}$ have such an element.

Proof of Theorem 5. Let $\mathfrak{i}$ represent $\langle\subseteq$; $:$. Let $U=\{u, v\}$ be a two element set disjoint from $A$. Set $A^{\prime}=A \cup U$. Extend each $f \in F$ to $A^{\prime}$ by setting $f\left(x_{0}, \cdots, x_{n-1}\right)=u$ if there is an $x_{i} \in U$. Let $u, v$ each be the value of a nullary operation. Define a unary operation $p$ and a binary operation $g$ as follows:

$$
\begin{aligned}
& p(x)=\left\{\begin{array}{l}
x, \text { if } x \in A ; \\
v, \text { if } x=u ; \\
u, \text { if } x=v ;
\end{array}\right. \\
& g(x, y)=\left\{\begin{array}{l}
x, \text { if } x, y \in A \text { or if } y=u ; \\
y, \text { if } x=u ; \\
v, \text { if } x \text { or } y=v .
\end{array}\right.
\end{aligned}
$$

Let $\mathfrak{V}^{\prime}=\left\langle A^{\prime} ; F \cup\{p, g, u, v\}\right\rangle$. For each $\varphi \in E(\mathfrak{l})$ define $\bar{\varphi}$ on $A^{\prime}$ by $x \bar{\varphi}=x \varphi$ if $x \in A$ and $x \bar{\varphi}=x$ if $x \in U$. For each $\theta \in \mathscr{C}(\mathfrak{O})$ set $\bar{\Theta}=\Theta \cup \omega_{U}$.

$\varphi \rightarrow \bar{\varphi}$ is an isomorphism from $\left(\mathfrak{S}(\mathfrak{V})\right.$ onto $\mathfrak{S}\left(\mathfrak{U}^{\prime}\right) . \quad \Theta \rightarrow \bar{\Theta}$ is an 
embedding of $\mathbb{C}(\mathfrak{U})$ into $\mathfrak{C}\left(\mathfrak{U}^{\prime}\right) . \quad \mathscr{C}\left(\mathfrak{U}^{\prime}\right)=\{\bar{\Theta} \mid \Theta \in \mathscr{C}(\mathfrak{U})\} \cup\left\{\mathfrak{c}_{A^{\prime}}\right\} . \quad$ The details are almost identical to the details in [3].

Proof of Theorem 8. Let $\mathfrak{A}_{0}=\left\langle A_{0} ; F_{0}\right\rangle$ and $\mathfrak{A}_{1}=\left\langle A_{1} ; F_{1}\right\rangle$ be algebras with $\mathfrak{F}\left(\mathfrak{U}_{i}\right)$ isomorphic to $\mathfrak{S}_{i}$ and $\mathfrak{E}\left(\mathfrak{U}_{i}\right)$ isomorphic to $\mathfrak{L}_{i}$. Assume $A_{0} \cap A_{1}=\varnothing$. Let $A_{2}=A_{0} \cup A_{1} \cup\{u, v\}$ where $u \neq v$ and $u$, $v \notin A_{0} \cup A_{1}$. Let $x_{0}, \cdots, x_{n-1} \in A_{2}$, and let $f \in F_{i}$. Extend $f$ to $A_{2}$ by setting $f\left(x_{0}, \cdots, x_{n-1}\right)=u$ if there exists $x_{j} \notin A_{i}$. Let $a_{i} \in$ $A_{i}$ and define two unary operations $g_{0}$ and $g_{1}$ by $g_{0}\left(a_{0}\right)=g_{0}(u)=u$ and $g_{0}\left(a_{1}\right)=g_{0}(v)=v$ and $g_{1}\left(\alpha_{i}\right)=a_{i}$ and $g_{1}(u)=v$ and $g_{1}(v)=u$. Define a binary $\mathrm{g}_{2}$ on $A_{2}$ by setting $g_{2}(x, v)=g_{2}(v, x)=x$ and $g_{2}(x, y)$ $=u$ otherwise. Take each of $u$ and $v$ as the value of a nullary operation. Let

$$
\mathfrak{A}_{2}=\left\langle A_{2} ; F_{0} \dot{\cup} F_{1} \dot{\cup}\left\{g_{0}, g_{1}, g_{2}, u, v\right\}\right\rangle=\left\langle A_{2} ; F_{2}\right\rangle \text {. }
$$

For each $\psi=\left\langle\varphi_{0}, \varphi_{1}\right\rangle \in E\left(\mathfrak{U}_{0}\right) \times E\left(\mathfrak{A}_{1}\right)$ define a mapping $\bar{\psi}$ on $A_{2}$ by $x \bar{\psi}=x \varphi_{i}$ if $x \in A_{i}$ and $x \bar{\psi}=x$ if $x=u$ or $x=v$. For each $\Phi=$ $\left\langle\Theta_{0}, \Theta_{1}\right\rangle \in \mathscr{C}\left(\mathfrak{U}_{0}\right) \times \mathscr{C}\left(\mathfrak{U}_{1}\right)$ set $\bar{\Phi}=\Theta_{0} \cup \Theta_{1} \cup \omega_{U}$. To complete the proof, one would show that $\mathscr{C}\left(\mathfrak{N}_{2}\right)=\left\{\bar{\Phi} \mid \bar{\Phi} \in \mathscr{C}\left(\mathfrak{R}_{0}\right) \times \mathscr{C}\left(\mathfrak{N}_{1}\right)\right\} \cup\left\{\iota_{A_{2}}\right\}$, that $\Phi \rightarrow$

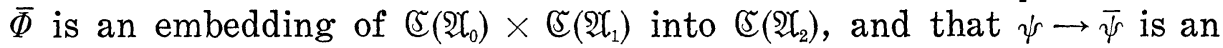
isomorphism of $\mathfrak{F}\left(\mathfrak{N}_{0}\right) \times \mathfrak{F}\left(\mathfrak{N}_{1}\right)$ onto $\mathfrak{F}\left(\mathfrak{U}_{2}\right)$. A few of the details follow.

Let $\sigma$ be an endomorphism of 2 . Note that $x \sigma=x$ for $x=u$ or $x=v$ since $u$ and $v$ are the vaules of nullary operations. Let $a_{i} \in A_{i}$. Now $g_{0}\left(a_{0} \sigma\right)=g_{0}\left(a_{0}\right) \sigma=u \sigma=u$. Thus, $a_{0} \sigma \in A_{0}$ or $a_{0} \sigma=u$. Suppose $a_{0} \sigma=u$. Then $u=a_{0} \sigma=g_{1}\left(a_{0}\right) \sigma=g_{1}\left(a_{0} \sigma\right)=g_{1}(u)=v$. Since $u \neq v$, it follows that $a_{0} \sigma \in A_{0}$. Similarly, $a_{1} \sigma \in A_{1}$. Thus, $\sigma=\overline{\left\langle\left.\sigma\right|_{A_{0}}, \sigma_{A_{1}}\right\rangle}$.

Suppose $a_{i} \in A_{i}$ and $a_{0} \equiv a_{1}(\Psi)$ and suppose $\Psi \in \mathscr{C}\left(\mathfrak{U}_{2}\right)$. Then $u \equiv$ $v(\Psi)$ since $u=g_{0}\left(a_{0}\right), v=g_{0}\left(a_{1}\right)$ and $g_{0}\left(a_{0}\right) \equiv g_{0}\left(a_{1}\right)(\Psi)$. For $x \in A_{0} \cup A_{1}$ it happens that $x \equiv u(\psi)$ iff $x \equiv v$ because $g_{1}(x)=x, g_{1}(u)=v$ and $g_{1}(v)=$ $u$. So if $x \in A_{0} \cup A_{1}$ and $y \in\{u, v\}$ and $x \equiv y(\Psi)$, then $u \equiv v(\Psi)$. If $u \equiv v(\Psi)$, then $\Psi=\iota_{A_{2}}$ because for any $x \in A_{2}, x \equiv u(\Psi)$. (This is because $u=g_{2}(u, x)$ and $\left.x=g_{2}(v, x) \cdot\right)\left.\Psi\right|_{A_{i}} \in \mathscr{C}\left(\mathfrak{U}_{i}\right)$. Thus, if $\Psi \neq \iota_{A_{2}}$, then $\Psi=\bar{\Phi}$ for some $\bar{\Phi} \in \mathscr{C}\left(\mathfrak{U}_{0}\right) \times \mathscr{C}\left(\mathfrak{U}_{1}\right)$, namely, for $\bar{\Phi}=\left\langle\left.\Psi\right|_{A_{\vartheta}},\left.\Psi\right|_{A_{1}}\right\rangle$.

Proof (of Theorem 6). Let $\mathfrak{U}_{i}=\left\langle A_{i} ; F_{i}\right\rangle$, for $i=0,1$, be algebras with $A_{0} \cap A_{1}=\varnothing$. We shall prove the theorem by showing that $\left\langle\mathfrak{S}\left(\mathfrak{U}_{0}\right), \mathfrak{S}\left(\mathfrak{N}_{0}\right)+\mathfrak{S}\left(\mathfrak{U}_{1}\right)+\mathfrak{S}_{1}\right\rangle$ and $\left\langle\mathfrak{S}\left(\mathfrak{A}_{1}\right), \mathfrak{S}\left(\mathfrak{U}_{0}\right)+\mathfrak{C}\left(\mathfrak{U}_{1}\right)+\mathfrak{C}_{1}\right\rangle$ are representable. First we consider the case with $\mathfrak{F}\left(\mathfrak{U}_{0}\right)$.

Let $u \neq v$ and $u, v \notin A_{0} \cup A_{1}$, and let $A_{2}=A_{0} \dot{\cup} A_{1} \dot{\cup}\{u, v\}$. For $f \in F_{i}$ extend $f$ to $A_{2}$ as in Theorem 8. Define the unary operations $g_{0}, g_{1}$ as in the proof of Theorem 8. Define the binary operation $g_{2}$ by setting $g_{2}\left(a_{0}, v\right)=g_{2}\left(v, a_{0}\right)=a_{0}$ for $a_{0} \in A_{0}$ and $g_{2}(x, y)=u$ otherwise. Let $a_{0} \in A_{0}$ and $a_{1}, b_{1} \in A_{1}$. Define the binary operation $g_{3}$ by $g_{3}\left(a_{1}, b_{1}\right)=$ 
$v$, if $a_{1} \neq b_{1}$, and $g_{3}(x, y)=u$ otherwise. Define the binary operation $g_{4}$ by $g_{4}\left(a_{1}, y\right)=y$ and $g_{4}(x, y)=u$ otherwise. Take each member of $A_{1} \cup\{u, v\}$ as the value of nullary operations. Set

$$
\mathfrak{N}_{2}=\left\langle A_{2} ; F_{0} \dot{\cup} F_{1} \dot{\cup}\left\{g_{0}, \cdots, g_{4}\right\} \dot{\cup}\{u, v\} \dot{\cup} A_{1}\right\rangle=\left\langle A_{2} ; F_{2}\right\rangle .
$$

For each $\varphi \in E\left(\mathfrak{2}_{0}\right)$ let $\bar{\varphi}$ be defined by $x \bar{\varphi}=x \varphi$ if $x \in A_{0}$ and $x \bar{\varphi}=x$ if $x \notin A_{0}$. For each $\Theta \in \mathscr{C}\left(\mathfrak{U}_{0}\right)+\mathscr{C}\left(\mathfrak{U}_{1}\right)$ define $\bar{\Theta}$ by $\bar{\Theta}=$ $\Theta \cup \omega_{A_{1}} \cup \omega_{\{u, v\}}$ if $\Theta \in \mathscr{C}\left(\mathfrak{U}_{0}\right)$ and $\bar{\Theta}=\Theta \cup \iota_{A_{0} \cup\{u, v\}}$ if $\in \mathscr{C}\left(\mathfrak{S i}_{1}\right)$.

To complete the proof for this pair, one would show that $\varphi \rightarrow \bar{\varphi}$ is an isomorphism of $\left(\mathfrak{F}\left(\mathfrak{N}_{0}\right)\right.$ onto $\mathfrak{F}\left(\mathfrak{A}_{2}\right)$, that $\Theta \rightarrow \bar{\Theta}$ is an embedding of $\mathbb{S}\left(\mathfrak{U}_{0}\right)+\mathbb{E}\left(\mathfrak{U}_{1}\right)$ into $\mathfrak{S}\left(\mathfrak{U}_{2}\right)$, and that $\mathscr{C}\left(\mathfrak{U}_{2}\right)=\left\{\bar{\Theta} \mid \Theta \in \mathscr{C}\left(\mathfrak{U}_{0}\right)+\mathscr{C}\left(\mathfrak{U}_{1}\right)\right\} \cup$ $\left\{\varepsilon_{A_{2}}\right\}$. A few of the details follow.

As in the proof of Theorem 8, for $\sigma \in E\left(\mathfrak{H}_{2}\right), A_{0} \sigma \subseteq A_{0}$ Clearly $x \sigma=x$ for $x \in A_{2}-A_{0}$ since every element is a nullary constant.

Let $\Theta \in \mathscr{C}\left(\mathfrak{A}_{2}\right)$. For $x \in A_{2}, x \equiv u$ iff $x \equiv v$ as in the proof of Theorem 8. Let $a_{i}, b_{i} \in A_{i}$. If $u \equiv v(\Theta)$, then $a_{0} \equiv u$ because $g_{2}\left(u, a_{0}\right)=u$ and $g_{2}\left(v, a_{0}\right)=a_{0}$. If $a_{1} \neq b_{1}$ and $a_{1} \equiv b_{1}(\Theta)$, then $u \equiv v$ because $g_{3}\left(a_{1}, b_{1}\right)=u$ and $g_{3}\left(b_{1}, b_{1}\right)=v$. Let $x \in A_{2}-A_{1}$ and let $z \in A_{2}$. If $a_{1} \equiv x(\Theta)$, then $z \equiv u(\Theta)$ because $g_{4}\left(a_{1}, z\right)=z$ and $g_{4}(x, z)=u$.

We now turn to considering the case for $\left\langle\mathfrak{F}\left(\mathfrak{A}_{1}\right)\right.$, $\mathfrak{S}\left(\mathfrak{U}_{0}\right)+\mathfrak{E}\left(\mathfrak{A}_{1}\right)+$ $\left.\mathfrak{C}_{1}\right\rangle$. We may now assume without loss of generality that $\mathfrak{F}\left(\mathfrak{H}_{0}\right)$ is the one element group and that there are no nullary operations in $\mathfrak{2}_{0}$. That this assumption can be made is verified in [6] and [7].

Let $w, r, s \notin A_{2}$. Let $A_{3}=A_{2} \cup\{w, r, s\}$. For $f \in F_{0}$ or $F_{1}$, change the value of $f\left(x_{0}, \cdots, x_{n-1}\right)$ to $w$ where in the above case it was $u$, i.e., in the case when there is an $x_{i}$ not an element of the appropriate $A_{i}$. Extend the $g_{i}$ in the following way: $g_{0}(r)=g_{0}(s)=v ; g_{0}(w)=w$; $g_{1}(w)=w ; g_{1}(r)=r ; g_{1}(s)=s$; still keep $g_{4}\left(a_{1}, y\right)=y$, but let $g_{4}(x, y)=$ $w$ otherwise; keep $g_{3}\left(a_{1}, b_{1}\right)=v$ for $a_{1} \neq b_{1}$ and $g_{3}(x, y)=u$ otherwise except let $g_{3}(w, w)=w ; g_{2}(w, q)=g_{2}(v, w)=g_{2}(w, w)=w$ and $g_{2}(x, y)=$ $u$ for any other new pair. Define three new operations as follows. Let $x, y \in A_{0}, z \in A_{0} \cup\{u, v, w\}$ and let $a_{1} \in A_{1}$. Set $g_{5}(w, w)=w, g_{5}(w, x)=$ $u, g_{5}(y, x)=v, g_{5}\left(z_{1}, z\right)=a_{1}$ and $g_{5}\left(z, a_{1}\right)=z$. Set $g_{6}(r)=s, g_{6}(s)=r$ and $g_{6}(x)=x$ otherwise. For $x, y \in A_{3}$ set $g_{7}(r, x)=g_{7}(x, r)=r$ and $g_{7}(x, s)=g_{7}(s, x)=x$, if $x \neq r$, and $g_{7}(x, y)=x$ otherwise. Take $w, r, s$ as values of nullary operations but don't take $A_{1} \cup\{u, v\}$ as nullaries. Set

$$
\mathfrak{T}_{3}=\left\langle A_{3} ; F_{0} \cup F_{1} \dot{\cup}\left\{g_{0}, \cdots, g_{7}\right\} \dot{\cup}\{w, r, s\}\right\rangle .
$$

For $\varphi \in E\left(\mathfrak{U}_{1}\right)$ define $\bar{\varphi}$ on $A_{3}$ as follows; $x \bar{\varphi}=x \phi$ if $x \in A_{1}, x \bar{\varphi}=$ $x$ if $x=w, r$, or $s$, and for $x \in A_{0} \cup\{u, v, w\}$ set $x \bar{\varphi}=x$ if $\varphi$ is $1-1$ and $x \bar{\varphi}=w$ if not. Let $\Theta \in \mathscr{C}\left(\mathfrak{U}_{0}\right)+\mathscr{C}\left(\mathfrak{H}_{1}\right)$. For $\Theta \in \mathscr{C}\left(\mathfrak{U}_{0}\right)$ set $\bar{\Theta}=$ $\Theta \cup \omega_{A_{1} \cup\{u, v, w, r, s\}}$, and for $\Theta \in \mathscr{C}\left(\mathfrak{U}_{1}\right)$ set $\bar{\Theta}=\Theta \cup \iota_{A_{0} \cup\{u, v, w\}} \cup \omega_{\{r, s\}}$. The 
outline of the rest of the proof is clear. Some details follow, particularly concerning endomorphisms.

Let $\theta \in \mathscr{C}\left(\mathfrak{N}_{3}\right)$. All the statements made about $\Theta$ in the previous case still hold with one change. Here if $a_{1} \in A_{1}$ and $x \in A_{3}-A_{1}$ and $x \equiv a_{1}$, then for all $z \in A_{3}, z \equiv w$ (instead of $u$ ). Some more should now be said. If $x \in A_{0}$ and $w \equiv x(\Theta)$, then $u \equiv v$ because $g_{5}(w, x)=$ $u$ and $g_{5}(x, x)=v$. If $u \equiv v(\Theta)$, then one gets $w \equiv u$ using $g_{2}$. Similar to the case with $u$ and $v$, for any $x \in A_{3}, x \equiv r$ iff $x \equiv r \equiv s$ (use $g_{6}$ ). Using $g_{7}$ we have that if $r \equiv s(\Theta)$ and $z \in A_{3}$, then $z \equiv r(\Theta)$.

Note that there can be no constant endomorphisms because there are three nullary operations with different values. Let $\sigma \in E\left(\mathfrak{U}_{3}\right)$. Let $x \in A_{0} \cup A_{1} \cup\{w\}$, and let $y \in A_{0} \cup A_{1} \cup\{u, v, w\}$. Using $g_{1}, x \sigma \neq u$ or $v$, and using $g_{6}, y \sigma \notin\{r, s\}$. Thus, $\left(A_{0} \cup A_{1} \cup\{w\}\right) \sigma \subseteq A_{0} \cup A_{1} \cup\{w\}$. Let $a_{i} \in A_{i}$. Now $w \sigma=w$. If $a_{1} \sigma=w$, then we would have $\sigma$ is a constant endomorphism because the congruence relation induced by $\sigma$ would be $\iota_{A_{3}}$. So $a_{1} \sigma \in A_{0} \cup A_{1}$. Now, as before, $A_{1} \sigma \cong A_{1}$. Similarly, one gets $\left(A_{0} \cup\{w\}\right) \sigma \subseteq A_{0} \cup\{w\}$. Using the congruence structure and the fact that $w \sigma=w$, either $A_{0} \sigma \subseteq A_{0}$ or $\left(A_{0} \cup\{u, v, w\}\right) \sigma=$ $\{w\}$. Clearly, if $A_{0} \sigma \subseteq A_{0}$, then $a_{0} \sigma=a_{0}$. When $A_{0} \sigma \subseteq A_{0}$, using the congruence structure and $g_{2}$, one gets $u \sigma=u$ and $v \sigma=v$. Finally, the congruence structure requires that if $\sigma$ is not $1-1$ on $A_{1}$, then $\sigma$ must be constant on $A_{0} \cup\{u, v, w\}$. And if $\sigma$ is constant on $A_{0} \cup$ $\{u, v, w\}$, then $\sigma$ would have the value $w$ there.

6. Concerning $\left\langle\subseteq, \mathfrak{C}_{3}\right\rangle$. From $\S 4$ we know that a necessary condition for the representability of $\left\langle\subseteq, \mathcal{C}_{3}\right\rangle$ is that $\left|\left\{\varepsilon_{\rho_{s}} \mid s \in S\right\} \cup\{c\}\right| \leqq 3$.

A stronger condition is proved to be sufficient. The representability of $\left\langle\subseteq, \mathfrak{S}_{2}\right\rangle$ has been characterized [3] (or see the remarks after Lemma 1), and $\left\langle S_{S}, \mathfrak{S}_{2}\right\rangle$ is representable iff $\left|\left\{\varepsilon_{\rho_{s}} \mid s \in S\right\} \cup\{c\}\right| \leqq 2$.

The method for proving the next lemma is very similar to that in Lemma 1. Recall the definition of $\varepsilon_{\varphi}$.

Lemma 6. Let $\mathfrak{X}=\left\langle A ; F^{\top}\right\rangle=$ be an algebra, and let $\theta \neq \omega, \theta \in$ $\mathscr{C}(\mathfrak{Q})$. There is an algebra $\mathfrak{X}^{\prime}=\left\langle A ; F^{\prime}\right\rangle$ so that:

(i) $\left.\mathscr{S}^{\prime}(\mathfrak{U})=\mathscr{S}^{(\mathfrak{i}} \mathfrak{I}^{\prime}\right)$;

(ii) $\mathscr{C}\left(\mathfrak{U}^{\prime}\right)=\{\omega, \Theta, \iota\}$;

(iii) $\varphi \in E\left(\mathfrak{U}^{\prime}\right)$ iff $\varphi \in E(\mathfrak{X}), \varepsilon_{0}=\omega, \theta$, or 6 and following conditions are satisfied:

(a) if $\varepsilon_{\varphi}=\Theta$, then $\varepsilon_{\varphi^{2}}=\Theta$ or c;

(b) if $\varepsilon_{\varphi}=\omega$ and is is any map with $\varepsilon_{\psi}=\theta$, then $\varepsilon_{\varphi \circ \psi}=\theta$.

REMARK. Obviously, one could not improve upon condition (a), but perhaps a proof could be given with (b) changed to read" $\cdots$, then $\varepsilon_{0, \psi}=\omega, \Theta$ or $\iota . "$ Notice that all automorphisms are kept. 
Proof. Add one 4-ary operation $g$ defined as follows:

$$
g(x, y, u, v)=\left\{\begin{aligned}
& u, \text { if } x \neq y(\Theta), u \neq v(\Theta) \text { or } \\
& \text { if } x \equiv y(\Theta), u \equiv v(\Theta) \text { and } \\
& x \neq y, u \neq v \\
& v, \text { otherwise } .
\end{aligned}\right.
$$

Set $\mathfrak{U}^{\prime}=\langle A ; F \cup\{g\}\rangle$. Clearly, (i) holds.

Proving that $\Theta \in \mathscr{C}\left(\mathfrak{U}^{\prime}\right)$ involves only routine calculation. So let $\Phi \in \mathscr{C}\left(\mathfrak{U}^{\prime}\right)$ with $\omega \neq \Phi$. So there exist $x, y$ with $x \neq y$ and $x \equiv y(\Phi)$. Suppose $x \neq \equiv(\theta)$. We will show that $\Phi=$ c. Let $u \neq v$. First assume $u \neq \equiv(\Theta)$. Then $u=g(x, y, u, v) \equiv g(y, y, u, v)=v(\Phi)$. Now assume $u \equiv v(\Theta)$. Since $x \not \equiv y(\Theta)$, there is a $z \in\{x, y\}$ with $z \not \equiv u(\Theta)$ and $z \neq v(\Theta)$. From above $u \equiv z(\Phi)$ and $v \equiv z(\Phi)$. Thus, $\left.u \equiv v_{(}^{\prime} \Phi\right)$. So $\Phi=\imath$. Now suppose for every $u, v$, with $u \equiv v(\Phi)$ that $u \equiv v(\Theta)$. Thus, $\Phi \subseteq \theta$. (We are still assuming $\Phi \neq \omega$, that $x \neq y$, and that $x \equiv y(\Phi)$.) We will show that in this case $\Phi=\Theta$. Let $u \equiv v(\Theta)$ with $u \neq v$. Then $u=g(x, y, u, v) \equiv g(y, y, u, v)=v(\Phi)$. So $\Theta \subseteq \Phi$ and $\Theta=\Phi$. Thus $\mathscr{C}\left(\mathfrak{X}^{\prime}\right)=\{\omega, \Theta, \iota\}$ and (ii) holds.

Obviously, if $\varphi \in E\left(\mathfrak{U}^{\prime}\right)$, then $\varphi \in E(\mathfrak{U})$ and $\varepsilon_{\varphi} \in\{\varphi, \Theta, \iota\}$. Suppose $\varepsilon_{\varphi}=\Theta$. Since $\varphi^{2} \in E\left(\mathfrak{U}^{\prime}\right)$, then $\varepsilon_{\varphi^{2}}=\Theta$ or $\iota$.

It is a routine calculation to show that if $\varepsilon_{\varphi}=\iota$ and $\varphi \in E(\mathfrak{H})$, then $\varphi \in E\left(\mathfrak{U}^{\prime}\right)$.

Let $\varphi \in E(\mathfrak{U})$ with $\varepsilon_{\varphi}=\Theta$ and with $\varepsilon_{\varphi_{2}}=\Theta$ or $\iota$. Consider $g(x, y$, $u, v)$. There are two possibly troublesome cases. One is if $g(x, y, u, v)$ $=u$ and $g(x \varphi, y \varphi, u \varphi, v \varphi)=v \varphi$. The other is if $g(x, y, u, v)=v$ and $g(x \varphi, y \varphi, u \varphi, v \varphi)=u \varphi$. The latter is the easiest to dispense with. If $g(x \varphi, y \varphi, u \varphi, v \varphi)=u \varphi$ and $u \varphi \neq v \varphi$, then $x \varphi \neq y \varphi$. Thus, $x \not \equiv y(\Theta)$ and $u \neq \equiv(\Theta)$. So $g(x, y, u, v)=u$ and $g(x, y, u, v) \varphi=u \varphi$. So now assume $g(x \varphi, y \varphi, u \varphi, v \varphi)=v \varphi$ and $g(x, y, u, v)=u$. Thus, either $x \not \equiv y(\Theta)$ and $u \neq \equiv(\Theta)$ or $x \equiv y(\Theta)$ and $u \equiv v(\Theta)$. Suppose $x \neq \equiv y$ and $u \neq v$. Then $x \varphi \neq y \varphi$ and $u \varphi \neq v \varphi$. Now $x \varphi \equiv y \varphi(\Theta)$ iff $u \varphi \equiv v \varphi(\Theta)$. Indeed, suppose that $x \varphi \equiv y \varphi(\Theta)$. Then $(x \varphi) \varphi=(y \varphi) \varphi$ and $\varepsilon_{\varphi^{2}} \neq \Theta$. So by assumption $\varepsilon_{\varphi^{2}}=\iota$. Thus, $(u \varphi) \varphi=(v \varphi) \varphi$, and $u \varphi \equiv v \varphi(\Theta)$. Similarly if $u \varphi \equiv v \varphi$, then $x \varphi \equiv y \varphi$. Thus, either $x \varphi \not \equiv y \varphi(\Theta), u \varphi \not \equiv$ $v \varphi(\Theta)$ or $x \varphi \equiv y \varphi(\Theta), u \varphi \equiv v \varphi(\Theta), x \varphi \neq y \varphi, u \varphi \neq v \varphi$. In any case, $g(x \varphi, y \varphi, u \varphi, v \varphi)=u \varphi \not \equiv v \varphi$. So $x \equiv y(\Theta)$ and $u \equiv v(\Theta)$. In this case $u \varphi=v \varphi$. Therefore, $g(x, y, u, v) \varphi=u \varphi=v \varphi=g(x \varphi, y \varphi, u \varphi, v \varphi)$. Thus, $\varphi \in E\left(\mathfrak{X}^{\prime}\right)$.

Suppose $\varphi$ is $1-1$ and $\varphi \in E\left(\mathfrak{U}^{\prime}\right)$. Let $\psi$ be any map with $\varepsilon_{\psi}=$ $\Theta$. Consider $\varepsilon_{\varphi \circ \psi}$. Suppose $\varepsilon_{\varphi \circ \psi}=\omega$. Then, $\varepsilon_{\psi} \neq \imath$. So there exist $x, y$ such that $x \neq \equiv(\Theta)$. Since $\Theta \neq \omega$, there exist $u$, $v$ with $u \neq v$ and $u \equiv v(\Theta)$. Since $u \neq v$, it follows that $(u \varphi) \psi \neq(v \varphi) \psi$. Thus, $u \varphi \not \equiv v \varphi(\Theta)$. Similarly, since $x \neq y, x \varphi \not \equiv y \varphi(\Theta)$. This implies that 
$g(x, y, u, v) \varphi=v \varphi \neq u \varphi=g(x \varphi, y \varphi, u \varphi, v \varphi)$. But since $\varphi$ is an endomorphism, we have that $\varepsilon_{\varphi \circ \psi} \neq \omega$. Suppose $\varepsilon_{\varphi \circ \psi}=\iota$. By a similar argument we would get that $\varepsilon_{\varphi \circ \psi} \neq \iota$ unless $\Theta=\iota$. So $\varepsilon_{\varphi \circ \psi}=\theta$.

Let $\varphi \in E(\mathfrak{X})$ with $\varepsilon_{\varphi}=\omega$. Let $\psi$ be any map with $\varepsilon_{\psi}=\theta$.

Suppose $\varepsilon_{\varphi \circ \psi}=\Theta$. Routine computation shows that $\varphi \in E\left(\mathfrak{Y}^{\prime}\right)$. The crucial point in these computations is that the assumption $\varepsilon_{\varphi \circ \psi}=$ $\Theta$ implies $\varphi$ presves both $\Theta$ and not- $\Theta$. Therefore (iii) holds.

Recall that if $\widetilde{S}=\langle S ; \cdot\rangle$ is a semigroup with identity, then $\mathscr{K}=$ $\left\{\varepsilon_{\rho_{s}} \mid \in S\right\}$. $\mathscr{L}(\mathfrak{S})$ is the algebra of left multiplications. $E(\mathfrak{L}(\mathfrak{S}))=$ $\left\{\rho_{s} \mid s \in S\right\}$.

THEOREM 9. Let $\mathfrak{\subseteq}=\langle S ; \cdot\rangle$ be a semigroup with identity.

(A) If $\left\langle S_{;} ; \mathfrak{S}_{3}\right\rangle$ is representable, then $|\mathscr{K} \cup\{c\}| \leqq 3$.

(B) If $|\mathscr{K} \cup\{c\}| \leqq 3$ and if for right cancellative $r$ and for $m$ that is neither right cancellative nor a right zero $r \cdot m$ is also neither right cancellative nor a right zero, then $\left\langle S_{5} ; \mathfrak{G}_{3}\right\rangle$ is representable.

REMARK. If $|\mathscr{K} \cup\{\ell\}|=2$, the rest of (B) holds trivially. So the sufficient condition includes all those representable pairs derived from Corollary 2 to Theorem 5 .

Proof. For part (A) see the corollary to Theorem 3.

Suppose the hypotheses of (B) hold. If $|\mathscr{K} \cup\{c\}|=2$, then $\left\langle\mathscr{S}_{;} \mathfrak{C}_{2}\right\rangle$ is representable. By Theorem 5, $\left\langle\Im_{;} ; \mathfrak{S}_{3}\right\rangle$ is representable. Suppose then that $\mathscr{C} \cup\{\iota\}=\{\omega, \Theta, \iota\}$ and that $\omega \neq \Theta \neq \iota$. Suppose $\varepsilon_{\rho_{m}}=\Theta$. Since

$$
\varepsilon_{\left(\rho_{m}\right)}{ }^{2}=\varepsilon_{\rho_{m 2}},
$$

it follows that

$$
\varepsilon_{\left(\rho_{m}\right)}{ }^{2}=\Theta \text { or } \iota \text {. }
$$

Let $\varepsilon_{\rho_{r}}=\omega$ and $\varepsilon_{\rho_{m}}=\Theta$. Since $r \cdot m$ is neither right cancellative nor a right zero, it follows that

$$
\varepsilon_{\rho_{r} \circ \rho_{m}}=\varepsilon_{\rho_{r m}}=\Theta
$$

Now apply Lemma 6 to $\Theta$ and $\mathcal{L}(\subseteq)$.

7. $\left\langle\mathfrak{S}_{,} \mathfrak{\Im}_{2}\right\rangle$ for unary algebras. In [3] G. Grätzer characterized the endomorphism semigroups of simple algebras. He also showed that not all such semigroups were isomorphic to endomorphism semigroups of simple unary algebras. Since previous representations involving congruence lattices and endomorphism semigroups had needed 
only unary algebras, he raised the question, "What semigroups are isomorphic to the endomorphism semigroups of simple unary algebras?" The answer to that question is that there are hardly any such semigroups.

Every endomorphism $\varphi$ induces a congruence relation which we have denoted by $\varepsilon_{\varphi}$. The difference with unary algebras is that every endomorphism also induces another congruence. Throughout $\mathfrak{A}=\langle A$; $F>$ will denote a unary algebra. For $\varphi \in E(\mathfrak{U})$ and $x, y \in A$ set $x \equiv$ $y\left(\Theta_{\varphi}\right)$ iff there exist natural numbers $i, j$ such that $x \varphi^{i}=y \varphi^{j}\left(x \varphi^{0}=\right.$ $x) . \quad \Theta_{\varphi}$ is the "extra" congruence. To prove that the substitution property holds for $\Theta_{\varphi}$, one needs that each operation of $\mathfrak{U}$ is unary or nullary.

Lemma 7. If $\varphi$ is $1-1$ and $\Theta_{\varphi}=\omega$ or $\iota$, then $\varphi$ is onto or $A=$ $\left\{a \varphi^{n} \mid n=0,1 \cdots\right\}$ for some $a \in A$.

Proof. $x \equiv x \Phi^{n}\left(\Theta_{\varphi}\right)$ for any natural number $n$ (by using the numbers $n, 0)$. In particular $x \equiv x \varphi\left(\Theta_{\varphi}\right)$. Thus, if $\Theta_{\varphi}=\omega$, then $x=$ $x \varphi$, and therefore, $\varphi$ is the identity map. Therefore, we can assume $\Theta_{\varphi}=\iota$, and this implies $x \equiv y\left(\Theta_{\varphi}\right)$ for any $x, y \in A$. Thus, for some $i$, $j, x \varphi^{i}=y \varphi^{j}$. If $i \leqq j$, then since $\varphi$ is $1-1$, we have that $x=y \varphi^{i-j}$. If $j \leqq i$, then $y=x \varphi^{i-j}$. Thus, $x \in\left\{y \varphi^{n} \mid n=0,1, \cdots\right\}$ or $y \in\left\{x \varphi^{n} \mid n=\right.$ $0,1, \cdots\}$. Suppose $\varphi$ is not onto. Then there is an $a$ such that $a \neq$ $x \varphi$ for all $x \in A(x \neq a)$. Thus, $a \notin\{x \varphi \mid n=0,1, \cdots\}$ for any $x \in A(x \neq$ a). Now since $x \in\left\{a \varphi^{n} \mid n=0,1, \cdots\right\}$ or $a \in\left\{x \varphi^{n} \mid n=0,1, \cdots\right\}$ for all $x \in A$, we have $x \in\left\{a \varphi^{n} \mid n=0,1, \cdots\right\}$ for all $x \in A$.

LEMmA 8. If $\Theta_{\varphi}=\omega$ or 6 and $\Theta$ is $1-1$ but not onto, then $\mathfrak{A}$ is not simple.

Proof. By Lemma 7, $A=\left\{a \varphi^{n} \mid n=0,1 \cdots\right\}$ for some $a \in A$. For $n>1, a \varphi^{n}=\left(a \varphi^{n-1}\right) \varphi$. Since $\varphi$ is not onto $a \neq x \varphi$ for any $x \in A$. Suppose $a \varphi^{i}=a \varphi^{j}$ and $i \neq j$. We may assume $i<j$. Since $\varphi$ is $1-$ $1, a=a \varphi^{j-i}$. Since $j-i \geqq 1, a=\left(a \varphi^{j-i-1}\right) \varphi$. Thus, $a \varphi^{i} \neq a \varphi^{j}$ if $i \neq$ $j$. Now set $E=\left\{a \varphi^{n} \mid n=0,2,4, \cdots\right\}$ and $D=\left\{a \varphi^{n} \mid n=1,3,5, \cdots\right\}$. By the above, $D \cap E=\varnothing$. Clearly, $D \cup E=A$. Let $\Phi$ be the equivalence relation whose only two classes are $D$ and $E . \Phi$ is a congruence. Since $\omega \neq \Phi \neq \imath, \mathfrak{U}$ is not simple.

For a simple algebra $\mathfrak{A}$ any right zero of $\mathfrak{F}(\mathfrak{U})$ is necessarily a constant mapping (unless $\mathfrak{\Im}(\mathfrak{U})$ is the one element group). See $\S 4$.

CoROllary. If $\mathfrak{X}$ is a simple unary algebra, then $E(\mathfrak{H})$ consists of automorphisms and constant mappings. 
G. Grätzer [3] characterized the automorphism group of a simple unary algebra as a cyclic group of order $p$ where $p=1$ or $p$ is a prime number. He also showed that if $p \neq 1$, then $A=\{\alpha \alpha \mid \alpha \in G(\mathfrak{U})\}$ for any $a \in A$.

LEMma 9. If $\mathfrak{X}$ is simple, $|G(\mathfrak{U})| \neq 1$, and there exists a right zero in $\mathfrak{S}(\mathfrak{U})$, then $|A|=2$ and $\mathfrak{S}(\mathfrak{U})=\left\langle A^{A} ; \circ\right\rangle$.

Proof. Let $\{a\}=A \varphi$. Let $f$ be an operation. Then $a=(f(a)) \varphi=$ $f(\alpha \varphi)=f(\alpha)$. If $x \in A$, then $x=\alpha \alpha$ for some $\alpha \in G(\mathfrak{X})$. Thus, $f(x)=$ $f(a \alpha)=f(a) \alpha=\alpha \alpha=x$. Therefore, $E(\mathfrak{X})=A^{A}$ and all equivalence relations are congruence relations. $|G(\mathfrak{U})| \geqq 2$ implies $|A| \geqq 2$. If $|A|>$ 2 , then there are more than two equivalence relations on $A$. Thus $|A|=2$.

Lemma 10. If $\mathfrak{X}$ is simple and $|G(\mathfrak{U})|=1$, then $|E(\mathfrak{U})| \leqq 2$.

Proof. Suppose there exist two constant endomorphisms $\varphi_{0}, \varphi_{1}$. Let $\left\{a_{0}\right\}=A \varphi_{0}$ and $\left\{a_{1}\right\}=A \varphi_{1}$. As in the proof of Lemma 3, $f\left(a_{0}\right)=$ $a_{0}$ and $f\left(a_{1}\right)=a_{1}$ for any operation $f$. If $|A|$ were two, then every operation would be the identity function and $|G(\mathfrak{H})|=2$. Thus, $|A|>$ 2. Set $x \equiv y(\Phi)$ iff $x=y$ or $x, y \in\left\{a_{0}, a_{1}\right\}$. Since every operation restricted to $\left\{a_{0}, a_{1}\right\}$ is the identity function, $\Phi$ is a congruence. Since $|A|>2, \Phi \neq \imath$. Since $\Phi \neq \omega, \mathfrak{A}$ is not simple.

THEOREM 10. Let $\mathfrak{S}=\langle S ; \cdot\rangle$ be a semigroup with identity. $\mathfrak{S}$ is isomorphic to the endomorphism semigroup of a simple unary algebra (i.e., $\left\langle\mathfrak{S}, \mathfrak{C}_{2}\right\rangle$ is representable by a unary algebra) if and only if $\mathfrak{S}$ is one of the semigroups listed below:

(i) the group of order $p, p=1$ or $p$ is a prime;

(ii) the two element semi-lattice;

(iii) a four element semigroup isomorphic to $\left\langle A^{A}\right.$; ० $\rangle$ where $|A|=2$. Moreover, if $\left\langle\subseteq, \mathbb{S}_{2}\right\rangle$ is representable by a unary algebra and $|S| \neq$ 1 , then $\left\langle\mathfrak{S}_{,} \mathfrak{S}_{2}\right\rangle$ is representable using a unary algebra with one operation.

Proof. It follows from the corollary to Lemma 8 and Lemmas 9 and 10 that the endomorphism semigroup of a simple unary algebra is one of those listed in (i) - (iii).

To complete the proof, we will represent $\left\langle\mathfrak{S}_{,} \mathfrak{F}_{2}\right\rangle$ for each $\subseteq$ listed in (i) - (iii).

In case $\mathfrak{S}$ is the one element group, let $A$ be a two element set. Set $\mathfrak{U}=\left\langle A ; A^{A}\right\rangle$. Clearly, $\mathfrak{U}$ has the required properties.

In case $\mathfrak{S}$ is $\left\langle A^{A} ; \circ\right\rangle$ where $|A|=2$, Let $\mathfrak{U}=\langle A ; F\rangle$ where $f$ is 
the identity map. Obviously, $\mathfrak{A}$ has the required properties.

In case $\mathfrak{S}$ is the two element semi-lattice, let $A=\{a, b\}$ with $a \neq$ b. Set $f(a)=f(b)=b$, and set $\mathfrak{X}=\langle A ; f\rangle$. Since $|A|=2, \mathfrak{X}$ is simple. The endomorphisms are exactly the identity map $\sigma$ and $\psi$ where $\psi=f$. Since $\sigma \circ \psi=\psi \circ \sigma=\psi=\psi \circ \psi$, the endomorphism semigroup is the two element semi-lattice.

In case $\subseteq$ is the group of order $p$ where $p$ is a prime, set $A=$ $\{0, \cdots, p-1\}$. Let $f(x)=x+1(\bmod p)$, and set $\mathfrak{U}=\langle A ; f\rangle$. Since $p$ is a prime it is easy to check that $\mathfrak{O}$ is simple. For $x \in A$ define the mapping $\varphi_{x}$ by $y \varphi_{x}=y+x$. Clearly, $x \rightarrow \varphi_{x}$ is an isomorphism from the cyclic group of order $p$ onto $\mathfrak{F}(\mathfrak{2})$.

\section{REFERENCES}

1. G. Birkhoff and O. Frink, Representations of lattices by sets, Trans. Amer. Math. Soc., 64 (1948), 229-316.

2. G. Grätzer, Universal Algebra, D. Van Nostrand Company, Princeton, New Jersey, 1968.

3. - On the endomorphism semigroup of simple algebras, Math. Annalen, 170 (1967), 334-338.

4. G. Grätzer and E. T. Schmidt, Characterizations of congruence lattices of abstract algebras, Acta. Sci. Math., 24 (1963), 34-59.

5. M. I. Gould and G. Grätzer, Boolean extensions and normal subdirect powers of finite universal algebras, Math. Z., 99 (1967), 16-25.

6. W. A. Lampe, On Related Structures of a Universal Algebra, thesis, 1969.

7. - On the congruence lattice characterization theorem, Trans. Amer. Math. Soc., (to appear).

8. - On the independence of certain related structures of a universal algebra, II, Alg. Univ. (to appear).

9. A. F. Pixley, The ternary discrimination function in universal algebra, (to appear).

10. E. T. Schmidt, Universale Algebren mit gegebenen Automorphismengruppen und Kongruenzverbanden, Acta. Math. Acad. Sci. Hungar., 15 (1964), 37-45.

11. - Universale Algebren mit gebebenen Automorphismengruppen und Unteralgebrenverbanden, Acta. Sci. Math., 24 (1963), 251-252.

12. H. Werner, A characterization of functionally complete algebras, Notices, Amer. Math. Soc., 17 (1970), 70T-A51.

Received June 10, 1971. The research for this paper was done when the author was a lecturer at the University of Manitoba. The results formed a portion of the author's dissertation at the Pennsylvania State University. The author wishes to thank Professor Grätzer for his many helpful suggestions concerning this paper.

UNIVERSITY OF HAWAII 



\section{PACIFIC JOURNAL OF MATHEMATICS}

\section{EDITORS}

\section{H. SAMElson}

Stanford University

Stanford, California 94305

C. R. Новву

University of Washington

Seattle, Washington 98105
J. DugunduI

Department of Mathematics University of Southern California

Los Angeles, California 90007

RICHARD ARENS

University of California

Los Angeles, California 90024

\section{ASSOCIATE EDITORS}
E. F. BECKENBACH
B. H. NeUmanN
F. WOLF
K. YosHIDA

\section{SUPPORTING INSTITUTIONS}

UNIVERSITY OF BRITISH COLUMBIA

UNIVERSITY OF SOUTHERN CALIFORNIA

CALIFORNIA INSTITUTE OF TECHNOLOGY

STANFORD UNIVERSITY

UNIVERSITY OF CALIFORNIA

UNIVERSITY OF TOKYO

MONTANA STATE UNIVERSITY

UNIVERSITY OF UTAH

UNIVERSITY OF NEVADA

WASHINGTON STATE UNIVERSITY

NEW MEXICO STATE UNIVERSITY

OREGON STATE UNIVERSITY

UNIVERSITY OF OREGON

OSAKA UNIVERSITY

UNIVERSITY OF WASHINGTON

* *

AMERICAN MATHEMATICAL SOCIETY

NAVAL WEAPONS CENTER 


\section{Pacific Journal of Mathematics}

\section{Vol. 43, No. $1 \quad$ March, 1972}

Alexander (Smbat) Abian, The use of mitotic ordinals in cardinal

arithmetic ....................................... 1

Helen Elizabeth. Adams, Filtrations and valuations on rings ......... 7

Benno Artmann, Geometric aspects of primary lattices .............. 15

Marilyn Breen, Determining a polytope by Radon partitions ........... 27

David S. Browder, Derived algebras in $L_{1}$ of a compact group .......... 39

Aiden A. Bruen, Unimbeddable nets of small deficiency .............. 51

Michael Howard Clapp and Raymond Frank Dickman, Unicoherent

compactifications ............................... 55

Heron S. Collins and Robert A. Fontenot, Approximate identities and the strict topology ................................... 63

R. J. Gazik, Convergence in spaces of subsets................. 81

Joan Geramita, Automorphisms on cylindrical semigroups ........... 93

Kenneth R. Goodearl, Distributing tensor product over direct product ..... 107

Julien O. Hennefeld, The non-conjugacy of certain algebras of

operators ................................... 111

C. Ward Henson, The nonstandard hulls of a uniform space ........... 115

M. Jeanette Huebener, Complementation in the lattice of regular

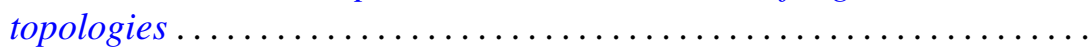

Dennis Lee Johnson, The diophantine problem $Y^{2}-X^{3}=A$ in a

polynomial ring .................................... 151

Albert Joseph Karam, Strong Lie ideals . . . . . . . . . . . . . . . . . . . . 157

Soon-Kyu Kim, On low dimensional minimal sets ............... 171

Thomas Latimer Kriete, III and Marvin Rosenblum, A Phragmén-Lindelöf

theorem with applications to $M(u, v)$ functions ..... . .

William A. Lampe, Notes on related structures of a universal algebra . . . . 189

Theodore Windle Palmer, The reducing ideal is a radical .

207

Kulumani M. Rangaswamy and N. Vanaja, Quasi projectives in abelian and module categories ................................ 221

Ghulam M. Shah, On the univalence of some analytic functions ......... 239

Joseph Earl Valentine and Stanley G. Wayment, Criteria for Banach

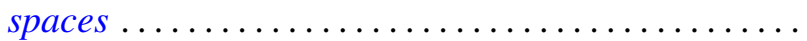

Jerry Eugene Vaughan, Linearly stratifiable spaces ............... 253

Zbigniew Zielezny, On spaces of distributions strongly regular with respect to partial differential operators ..................... 